\title{
Electronic and crystallographic structure of apatites
}

\author{
L. Calderín* and M. J. Stott \\ Department of Physics, Queen's University, Kingston, ON, Canada K7L 3N6
}

A. Rubio

Department of Material Physics, University of the Basque Country, Centro Mixto CSIC-UPV/EHU and Donostia International Physics Center (DIPC), 20018 Donostia-San Sebastian, Spain

(Received 23 December 2002; published 15 April 2003)

\begin{abstract}
An $a b$ initio study of four different stoichiometric apatites (oxyapatite, hydroxyapatite, fluorapatite, and chlorapatite) is presented. The calculations were performed using density-functional theory with the localdensity approximation for exchange and correlation, and a full relaxation of the electronic structure, the atomic arrangement, and the unit cell. Hexagonal unit cells were obtained for all four apatites, and the calculated atomic arrangements are in close agreement with observation in those cases for which the structure is firmly established. A zero-temperature structure is predicted for oxyapatite, and two possible configurations were found for the $\mathrm{Cl}^{-}$ions in chlorapatite. The possibility of the monoclinic structure in hydroxyapatite and chlorapatite was also studied but no indication of greater stability with respect to the hexagonal structure was found. A relationship between the structure of the apatites and that of pure calcium is discussed.
\end{abstract}

DOI: 10.1103/PhysRevB.67.134106

PACS number(s): 61.66.Fn, 71.20.Ps

\section{INTRODUCTION}

A material which actively participates in the naturally occurring bone replacement process of the body has been developed as thin films and in powder form. ${ }^{1}$ This material, a mixture of calcium phosphates, is obtained by adding silica or silicon to hydroxyapatite (HA) and firing it at $1000{ }^{\circ} \mathrm{C}$ or more. ${ }^{1}$ The bioactive component of the material seems to be $\alpha$-tricalcium phosphate stabilized at room temperature by $\mathrm{Si}$ doping. This component has been termed $\mathrm{Si}-\alpha$-TCP. To help gain understanding of how this phase mixture develops, and to shed light on the role of silicon and the origins of the unique bioactivity of this material, $a b$ initio quantummechanics based simulation methods are being used to investigate the atomic structure and to aid the interpretation of experimental data. A study of the calcium apatite group of materials, of which hydroxyapatite is a member, seems to be a mandatory first step to establish a reference for further studies on the considerably more complicated tricalcium phosphates.

Calcium apatites have the general chemical formula $\mathrm{Ca}_{5}\left(\mathrm{PO}_{4}\right)_{3} X$, where $X$ is an electronegative element or group such as a halogen or an $\mathrm{OH}$ group. The structure of the apatites can be viewed as consisting of unconnected $\mathrm{PO}_{4}^{3-}$ tetrahedra with $\mathrm{Ca}^{2+}$ in the space between and a column of $X^{-}$ions along the $c$ axis to balance the charge. The type and location of these anions distinguish the various calcium apatites. Two kinds of structure have been found experimentally depending on the stoichiometry, temperature, and pressure. ${ }^{2}$ These are the hexagonal and the monoclinic structures where the monoclinic unit cell is obtained from the hexagonal one by doubling the $b$ lattice parameter with an alternating arrangement of the anion chain. In the case of hydroxyapatite and chlorapatite the hexagonal structure is usually associated with nonstoichiometry, with the stoichiometric compound adopting the monoclinic structure. This situation highlights the difficulty in preparing stoichiometric apatites because of their great flexibility in accepting substitutions.

Fluorapatite (FA) is a mineral with chemical formula $\mathrm{Ca}_{5}\left(\mathrm{PO}_{4}\right)_{3} \mathrm{~F}$, which is usually taken to be a model for the apatites group. X-ray diffraction measurements ${ }^{2}$ at room temperature show a hexagonal unit cell containing two formula units with space group $P 6_{3} / \mathrm{m}$. Structural data for fluorapatite and the other apatites considered here are given in Table I. The $\mathrm{F}^{-}$ions lie at the center of the calcium triangles at $z=1 / 4$ and $z=3 / 4$. A possible phase transition to a monoclinic structure at around $130 \mathrm{~K}$ has been noticed but this has not been firmly established. ${ }^{2,3}$

Chlorapatite (CA), $\mathrm{Ca}_{5}\left(\mathrm{PO}_{4}\right)_{3} \mathrm{Cl}$, which is also a mineral is reported ${ }^{2,4}$ to have a monoclinic structure at room temperature with four formula units per unit cell. A hexagonal structure with two formula units per cell has been observed at temperatures above $310^{\circ} \mathrm{C}$. X-ray-diffraction studies initially located the $\mathrm{Cl}^{-}$ion at $z=0$ and $z=1 / 2$ in the hexagonal unit cell, but further investigation showed them as shifted from these positions. The monoclinic structure has the chlorine ions shifted up and down in alternate chains, whereas the fit of the hexagonal structure to the x-ray data retains the mirror plane and half occupies the resulting four chlorine site by the two atoms per cell.

Hydroxyapatite (HA), $\mathrm{Ca}_{5}\left(\mathrm{PO}_{4}\right)_{3} \mathrm{OH}$, is the main inorganic constituent of natural bone. In addition it is a naturally occurring but rare mineral. ${ }^{2}$ It is also reported ${ }^{2,5-7}$ to have a monoclinic structure with four formula units per unit cell, and a phase transition to a hexagonal structure around $370{ }^{\circ} \mathrm{C}$, but again the problem with the preparation of pure stoichiometric apatites could be influencing these results. The structure of the hexagonal phase of hydroxyapatite also presents a problem. Following failed attempts to fit x-raydiffraction data using the space group $P 6_{3}$, the structure was found with space group $P 6_{3} / m$ through the addition of two mirror planes perpendicular to the $z$ axis and passing through $z=1 / 4$ and $z=3 / 4$. However, this structure has four possible sites for the two $\mathrm{OH}$ groups per unit cell, a difficulty which 
TABLE I. Fully relaxed binding energies, Kohn-Sham band gaps, and unit-cell parameters of the apatites.

\begin{tabular}{|c|c|c|c|c|}
\hline \multirow{2}{*}{ Apatite } & \multirow{2}{*}{$\begin{array}{l}\text { Binding } \\
\text { energy } \\
(\mathrm{eV})\end{array}$} & \multirow{2}{*}{$\begin{array}{l}\text { Khon-Sham } \\
\text { gap } \\
(\mathrm{eV})\end{array}$} & \multicolumn{2}{|c|}{ Unit-cell parameters } \\
\hline & & & Calculated & Experimental \\
\hline FA & -267.9 & 5.9 & $\begin{array}{c}\text { S.G.: } P 6_{3} / m \\
a=b=9.06 \AA, c=6.84 \AA \\
\alpha=\beta=90.0^{\circ}, \gamma=120.0^{\circ}\end{array}$ & $\begin{array}{c}\text { S.G.: } P 6_{3} / m^{2} \\
a=b=9.398 \AA, c=6.878 \AA\end{array}$ \\
\hline CA & -263.3 & 5.6 & $\begin{array}{c}\text { S.G.: } P 6_{3} \\
a=b=92.5 \AA, c=6.79 \AA \\
\alpha=\beta=90.0^{\circ}, \gamma=120.0^{\circ}\end{array}$ & $\begin{array}{c}\text { S.G.: } P 6_{3} / m^{2} \\
a=b=9.628 \AA ⿻ \text { } c=6.764 \AA\end{array}$ \\
\hline HA & -280.6 & 5.4 & $\begin{array}{c}\text { S.G.: } p 6_{3} \\
a=b=9.11 \AA, c=6.86 \AA \\
\alpha=\beta=90.0^{\circ}, \gamma 120.0^{\circ}\end{array}$ & $\begin{array}{c}\text { S.G.: } P 6_{3} / m^{2} \\
a=b=9.422 \AA, c=6.885 \AA\end{array}$ \\
\hline $\mathrm{OA}$ & -262.2 & 4.0 & $\begin{array}{c}\text { S.G.: } P \overline{6} \\
a=b=9.06 \AA \text { 的 } c=6.73 \AA \\
\alpha=90.3^{\circ}, \beta=90.0^{\circ}, \gamma=119.9^{\circ}\end{array}$ & S.G.: $P \overline{6}^{10}$ \\
\hline c empty & -251.23 & & $\begin{array}{c}\text { S.G.: } P_{3} / m \\
a=b=9.10 \AA, c=6.82 \AA \\
\alpha=\beta=90.0^{\circ}, \gamma=120.1^{\circ}\end{array}$ & \\
\hline
\end{tabular}

was resolved by assuming 0.5 occupancy per site resulting from disorder in every anion column or disorder from column to column. ${ }^{8}$

The existence of oxyapatite, $\mathrm{Ca}_{5}\left(\mathrm{PO}_{4}\right)_{3} \mathrm{O}_{0.5}$, was in doubt for some time but it is now well established. ${ }^{9}$ However, its structure has not been completely clarified. A hexagonal crystal structure with lattice parameters close to those of hydroxyapatite has been suggested on the basis of highresolution electron microscopy ${ }^{10}$ following the on-beam decomposition of hydroxyapatite, but is not certain that the final product was oxyapatite.

The results of calculations on fluor-, chlor-, hydroxy-, and oxyapatite will be presented. In order to investigate the stability of the apatites against removal of the electronegative group $X$ we have also performed calculations on a defective apatite $\mathrm{Ca}_{5}\left(\mathrm{PO}_{4}\right)_{3}$ which we refer to as "c empty." The $a b$ initio method we have used involves a calculation for a fixed configuration of the ions of the total energy of electrons and ions, and the forces on the ions. The latter are used to relax the ions to new positions which lower the total energy. The process continues iteratively until the equilibrium arrangement of ions is reached at which the energy is minimized and the net force on the ions vanishes to desired precision. We have used density-functional theory for handling the exchange and correlation parts of the electron interactions, and first-principles pseudopotentials for the electron-ion interactions. In addition to the total energy and the equilibrium positions of the atoms within the unit cell, the equilibrium size and shape of the unit cell can also be obtained. In this initial study the final atomic arrangement and the lattice parameters are for static equilibrium.

The only $a b$ initio simulation of an apatite that we know of is the work on fluorapatite reported by Louis-Achille et al. ${ }^{11}$ Similar pseudopotentials to those we have employed and a plane-wave basis set were used to perform a relaxation of the atomic positions and unit-cell parameters but with the lattice constrained to be hexagonal. Semiempirical tightbinding calculations have been performed on hydroxyapatite by Song et al., ${ }^{12}$ but these were for observed atomic positions and do not shed any light on the structure.

This paper has four sections. The method is outlined in the next section. In the third section the results are presented and discussed, and conclusions are drawn in the final section.

\section{COMPUTATIONAL APPROACH}

Density-functional theory ${ }^{13}$ with the local-density approximation (DFT-LDA) provides the theoretical framework of our calculations. Due to the large number of electrons per unit cell in the apatites an approach based on pseudopotentials was adopted. ${ }^{14}$ The full ionic potential of the atoms was replaced by softer first-principles pseudopotentials which were generated in the Troullier-Martins manner. ${ }^{15}$ The exchange-correlation functional used the results of Ceperley and Alder ${ }^{16}$ as parametrized by Perdew and Zunger. ${ }^{17}$ The LDA usually overbinds and underestimates bondlengths and this is sometimes corrected by use of generalized gradient corrections (GGA) for exchange and correlation. However, we have chosen to use the LDA because there is a small saving in computational time, and, more importantly, the errors due to LDA although often larger than for the GGA are predictable and seem to preserve trends. The calculations employed the SIESTA code ${ }^{18}$ which uses a basis set of truncated atomic orbitals.

The pseudopotentials were generated and fully tested taking into account the presence of a large number of oxygen 
atoms in all the systems of interest. The dependence on environment was done by comparing all-electron and pseudopotential calculations for a large set of ionic configurations. Considerable effort was put into developing pseupotentials which minimized the number of electrons to be treated while avoiding, as much as possible, core overlap. The systems used for testing the $\mathrm{Ca}, \mathrm{P}, \mathrm{O}$, and $\mathrm{H}$ pseudopotentials were the solids $\mathrm{CaO}$ (lime), $\mathrm{P}_{2} \mathrm{O}_{5}, \mathrm{CaF}_{2}, \mathrm{CaCl}_{2}$, and the water molecule. The tests compared calculated atomic excitation energies and geometrical parameters, e.g., bond lengths, bond angles, unit-cell parameters, with those obtained from experiments and/or other calculations. During these tests the need for semicore states for calcium and nonlinear core corrections for phosphorus became clear. The pseudopotentials used in the calculations of the apatites were generated using the following reference configurations: $1 s^{1}$ for $\mathrm{H}, 2 s^{2} 2 p^{4}$ for $\mathrm{O}, 2 s^{2} 2 p^{5}$ for $\mathrm{F}, 2 s^{1} 2 p^{0.75} 3 d^{0.25}$ for $\mathrm{P}^{+3}, 3 s^{2} 3 p^{5}$ for $\mathrm{Cl}$, and $3 s^{2} 3 p^{6}$ for $\mathrm{Ca}^{+2}$. The core radii used were the following: 0.6 a.u. for $\mathrm{H}, 1.15$ a.u. for $\mathrm{O}, 1.20$ a.u. for $\mathrm{F}, 1.60$ a.u. for $\mathrm{P}^{+3}, 1.65$ a.u. for $\mathrm{Cl}$, and 1.80 a.u. for $\mathrm{Ca}$.

The basis sets used were of the DZP type: double $\zeta$ with polarization. The split-norm parameter used was 0.20 for all the atoms except $\mathrm{H}$ for which a split of 0.5 was used. ${ }^{18}$ The energy shifts which determine the cutoff radii of the basis set functions were around $200 \mathrm{meV}$.

The atomic positions and the unit-cell parameters were allowed to relax to the minimum energy configuration for all the apatites using a cutoff energy of 300 Ry. This cutoff controls the real space grid spacing used in calculation of the Hartree potential via fast Fourier transforms. ${ }^{19}$ Six $k$ points and a force tolerance of $0.05 \mathrm{eV} / \AA$ were used. Tests of the convergence with respect to the cutoff energy, the number of $k$ points, and the force tolerance showed that under the chosen computational conditions the convergence of the total energy is about $10 \mathrm{meV}$ per unit cell, while the errors in the unit-cell parameters were about $0.02 \AA$ for the unit-cell parameters lengths and $0.2^{\circ}$ for the angles. Vibrational and zero-point motion contributions to the total energy were not included in the calculations.

\section{RESULTS AND DISCUSSION}

For all the apatites considered a stable minimum-energy configuration with a hexagonal unit cell was found giving binding energies around $-260 \mathrm{eV}$ per formula unit and energy gaps between 4 and $5 \mathrm{eV}$. These energy gaps are valence-conduction band gaps of the Kohn-Sham energy levels. We recognize that they are expected to underestimate the gap in the excitation energy spectrum, calculated using, for example, the GW approximation. ${ }^{20}$ They are given here as additional measures of the stability of the materials.

The results for the binding energies and energy gaps, and the unit-cell parameters for fluorapatite (FA), chlorapatite (CA), hydroxyapatite (HA), and oxyapatite (OA), together with experimental crystallographic data as obtained from diffraction techniques are shown in Table I. The calculated crystallographic structure of FA, which apart from the arrangement of the anion $c$-axis chains, is typical of the others is illustrated in Fig. 1(a). The structure has two alternating
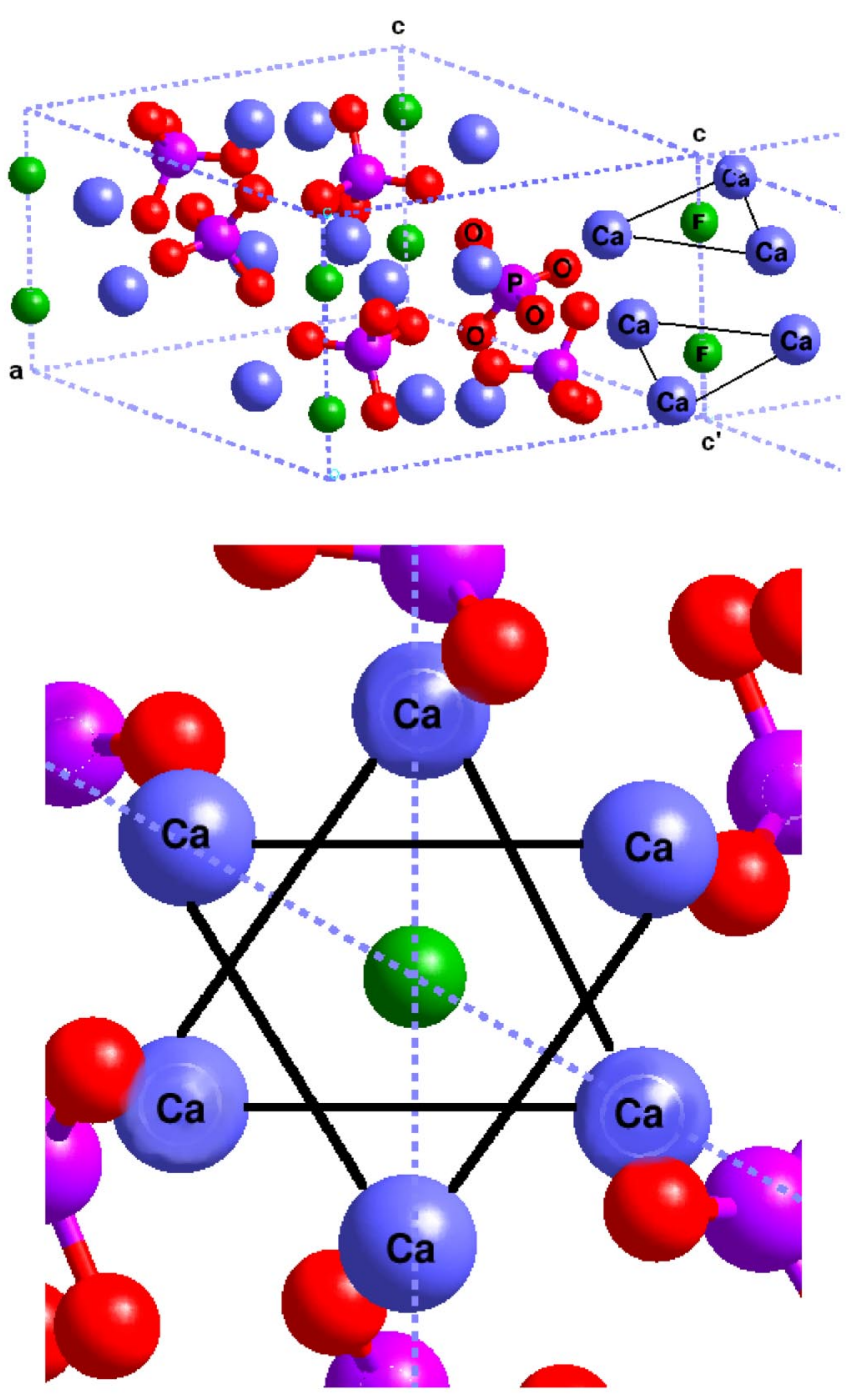

FIG. 1. (a) General view of the fluorapatite structure including calcium triangles. (b) Top view of four unit cells along $c-c^{\prime}$ showing the calcium triangles. The phosphorus triangles can also be seen.

layers perpendicular to the $c$ axis: one with only calcium atoms and the other one with calcium ions and $\mathrm{PO}_{4}$ groups. The anions occupy positions close to or on those planes depending on their chemical nature.

\section{A. Crystal structure}

The calculated lattice parameters are almost all smaller than the observed ones, the $a$ parameters uniformly smaller by about $3 \%$. Systematically smaller bond lengths often accompanied by overbinding is a characteristic of the localdensity approximation for exchange and correlation. However, observed trends are usually given well. For example, the calculated $a$ lattice parameter increases in the order OA, FA, HA, CA, a trend in the experimental results pointed out by Elliot. ${ }^{2}$ The observed trend in $c$ is also given except for the change from OA to FA. The calculated space groups of FA and OA are in agreement with experiment, ${ }^{2,10}$ but the stable position of the $c$-axis oxygen was found to be off the 
calcium triangle. In the cases of HA and CA the calculated structures do not contain the mirror planes of the $P 6_{3} / \mathrm{m}$ space group. Instead the $P \overline{6}$ space group was found. The calculations suggest that for stoichiometric HA a hexagonal unit cell is possible with all the $\mathrm{OH}$ groups oriented in the same direction. For CA we find that the chlorine anions are displaced from the $\mathrm{z}=1 / 2$ positions which also lowers the symmetry to the $P 6_{3}$ space group. Since the structures with the mirror planes fitted to the $\mathrm{x}$-ray data have four sites for the two OH's there must be imperfections in the samples due to disorder or impurities. The ab initio calculations of LouisAchille et al. on FA also using the LDA but with plane waves as a basis set gave unit-cell parameters: $a=9.375 \AA$ and $c=6.884 \AA$, which are in better agreement with experiment than our results. However, their calculations used one $k$ point only, whereas our tests indicate that six $k$ points are required for converged total energies. No other details of the crystal structure of FA were reported.

The lack of an inversion center in HA and CA suggests the theoretical possibility of piezoelectricity and/or ferroelectricity in these materials. In order to investigate this further we have performed a simulation of HA with the hexagonal unit cell doubled along $\mathbf{b}$ and containing four formula units. The calculations for these two systems, the hexagonal structure with all the OH's oriented in the same direction and the monoclinic one with the OH's oriented in the same direction along a column but in the opposite direction in the next column, resulted in indistinguishable total energies per molecule. For CA the monoclinic structure with four formula units per unit cell also has a very similar total energy per molecule to that of the hexagonal structure. This together with the apparent stability we have found for the apatite structure with the $c$ channel empty illustrates how flexible the apatites are in accommodating and allowing movement of anions of different kinds along the $c$ axis.

A feature in the results for the hexagonal structure of CA could have interesting consequences. We found energy minima for two different structures, the total energies being very similar. The structures had slightly different lattice parameters, and whereas in one the chlorine atoms are at the $z=1 / 2$ position in the hexagonal cell, in the other they are displaced along the $z$-axis from this position. We anticipate that these minima which are separated by a very low energy barrier could lead to interesting vibrational effects.

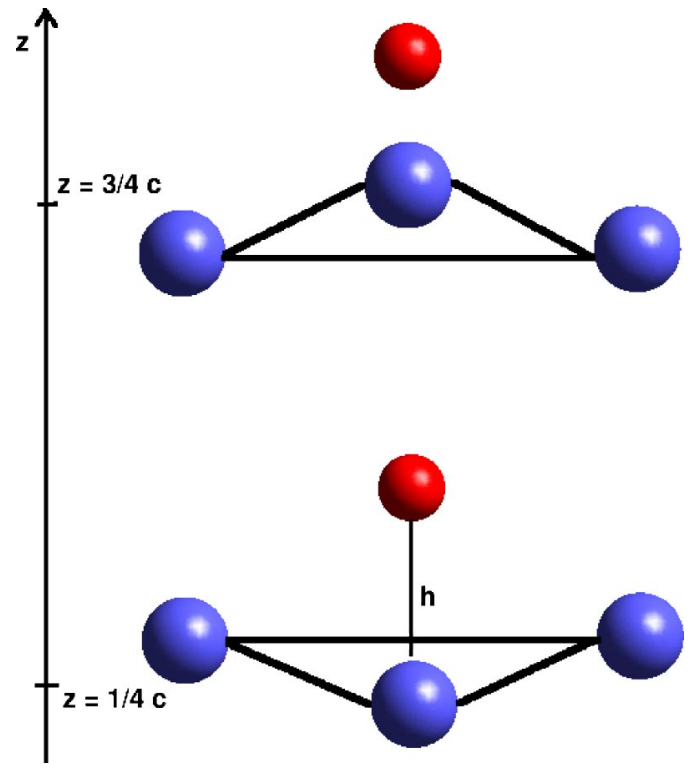

FIG. 2. Closeup of the region around the $c$ axis. The two calcium triangles rotated by $60^{\circ}$ and the anion are shown. For FA the $\mathrm{F}$ ion is at $z=1 / 4$ in the plane of the calcium triangles, for CA the $\mathrm{Cl}$ ion is close to the $z=0.5 c$ position, for HA the $\mathrm{OH}$ is just off the calcium triangles while for $\mathrm{OA}$ the $\mathrm{O}$ ion is at $z=0$.

\section{B. Atomic arrangement}

The apatite structures differ in the arrangement of the columns of anions along the $c$ axis. The $\mathrm{Ca}$ and $\mathrm{PO}_{4}$ ion respond little to the arrangement of the anion columns and the rest of the units are qualitatively very similar. The anion arrangement is conveniently characterized by the distance $h$ between the anions and the smallest $\mathrm{Ca}$ triangles which are perpendicular to the $c$ axis. The situation is illustrated in Fig. 2. There is good agreement between the calculated and observed values for $h$ which are given in Table II. In the case of the $\mathrm{OH}$ group in $\mathrm{HA}, h$ is measured from the position of the oxygen, the hydrogen is $0.9 \AA$ further away from the $\mathrm{Ca}$ triangle and also on the $c$ axis. The sizes of the $\mathrm{Ca}$ and $\mathrm{P}$ triangles are also listed in Table II; there is little variation for these apatites. The $\mathrm{PO}_{4}$ groups are very rigid displaying little variation in size and shape. The calculations yield an average P-O bond length of $1.56 \AA$ with a standard deviation less than $0.01 \AA$ for all the apatites. The corresponding experi-

TABLE II. Height $h$ of the anions with respect to the calcium triangle and length of the side of the smaller triangle perpendicular to the $c$ axis formed by calcium and phosphorus atoms (see Fig. 2).

\begin{tabular}{|c|c|c|c|c|c|c|}
\hline \multirow[t]{2}{*}{ Apatite } & \multicolumn{2}{|c|}{$\begin{array}{c}h \\
(\AA)\end{array}$} & \multicolumn{2}{|c|}{$\begin{array}{l}\text { Calcium } \\
\text { triangle } \\
(\AA)\end{array}$} & \multicolumn{2}{|c|}{$\begin{array}{c}\text { Phosphorus } \\
\text { triangle } \\
(\AA)\end{array}$} \\
\hline & Calc. & Expt. & Calc. & Expt. & Calc. & Expt. \\
\hline Fluorapatite & 0.01 & 0.00 & 3.85 & 3.99 & 5.97 & 6.25 \\
\hline Chlorapatite & 1.33 & 1.22 & 4.06 & 4.17 & 6.16 & 6.54 \\
\hline Hydroxyapatite & 0.27 & 0.3 & 4.00 & 4.08 & 6.02 & 6.27 \\
\hline Oxyapatite & 1.46 & & 3.67 & & 6.00 & \\
\hline$c$ empty & & & 3.82 & & 6.02 & \\
\hline
\end{tabular}


TABLE III. Standard deviation of the bond angle (in degrees) of $\mathrm{PO}_{4}$ groups located close to the border and around the center (interior) of the unit cell. The number of parentheses is the same standard deviation in percent with respect to the average. The average value is $109.4^{\circ}$ for all the $\mathrm{PO}_{4}$ groups for both calculated and experimental magnitudes.

\begin{tabular}{lcccc}
\hline \hline & \multicolumn{2}{c}{ Calculated } & \multicolumn{2}{c}{ Experimental } \\
Apatite & Border & Interior & Border & Interior \\
\hline Fluorapatite & $3.4(3.1)$ & $2.3(2.1)$ & $1.89(1.7)$ & $1.89(1.7)$ \\
Chlorapatite & $3.0(2.7)$ & $3.0(2.7)$ & $2.51(2.3)$ & $2.51(2.3)$ \\
Hydroxyapatite & $2.3(2.1)$ & $2.7(2.1)$ & $2.00(1.8)$ & $2.03(1.8)$ \\
Oxyapatite & $3.9(3.6)$ & $3.1(2.8)$ & & \\
\hline \hline
\end{tabular}

mental averages are 1.54 and $0.01 \AA$, respectively. The calculated average O-P-O angle is $109.4^{\circ}$ for all the apatites, with standard deviations ranging between $2.3^{\circ}$ and $3.4^{\circ}$. The deviation of the observed O-P-O angles is somewhat less: $1.9^{\circ}$ to $2.5^{\circ}$ (see Table III). The orientation of the $\mathrm{PO}_{4}$ groups is also in good agreement with experiment, with discrepancies in spherical polar angles being less than $3^{\circ}$ except for $\mathrm{CA}$, in which the simulations have the $\mathrm{PO}_{4}$ groups which form the triangle about the $c$-axis chain tilted nearer the $c$ axis by about $8^{\circ}$ with respect to the observed orientations.

We also note a similarity between the arrangement of the $\mathrm{Ca}$ atoms in the apatites, and that in the $\beta$ structure of pure calcium. $\beta-\mathrm{Ca}$ is hexagonal, and if the line of $\mathrm{Ca}$ atoms along the $c$ axis is removed the remaining arrangement consists of equilateral $\mathrm{Ca}$ triangles perpendicular to the $c$ axis with a Ca-Ca distance of $3.95 \AA$ which are very similar in size and arrangement to the $\mathrm{Ca}$ triangles around the $c$ axis in the apatites. Surrounding these are hexagons of $\mathrm{Ca}$ atoms, again, as in the apatites, which are in the latter case considerably expanded to accommodate the $\mathrm{PO}_{4}$ groups.

\section{Electronic structure}

Figure 3 shows the total densities of Kohn-Sham states of the different apatites. DFT-LDA that we have used is reported to reproduce the main features of the excitation spec-

\section{Density of States}

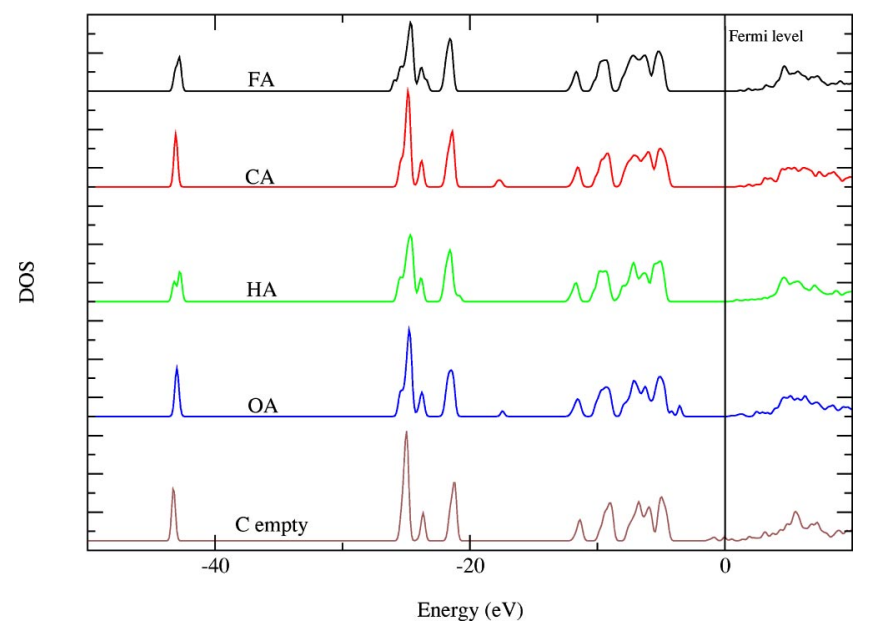

FIG. 3. Total density of states. trum in the region of the valence band and below, but underestimates the band gap and fails for the conduction band. ${ }^{20,21}$ Our interest in the density of states (DOS) is the light it sheds on the electronic structure of the ground state. Interpretation of the DOS is aided by densities of states projected onto a particular species of atom, which have also been calculated. Examples for $\mathrm{O}$ and for the anions in the $c$-axis chains are shown in Figs. 4 and 5, respectively. The main features of the total DOS's are common to all the apatites. The peak at $-43 \mathrm{eV}$ is due to $\mathrm{Ca} 3 p$ states. The roughly 7 -eV-wide band at about $-23 \mathrm{eV}$ and the valence bands consist of $\mathrm{P}$ and $\mathrm{O}$ states binding the $\mathrm{PO}_{4}$ groups with $\mathrm{Ca}$ states also contributing to the bottom of the lower bands. The energy gaps are between 5 and $6 \mathrm{eV}$ wide except for that of OA which at $4 \mathrm{eV}$ is much reduced. Although most of the main differences in the total DOS's are due directly to the anions in the $c$-axis chains, the splittings of the $\mathrm{Ca} 3 p$ peaks in FA and HA, absent in CA and OA, arise because of the location of the two distinct $\mathrm{Ca}$ sites with respect to the anions in the $c$-axis chains. In FA and HA the $\mathrm{Ca}$ site which forms the triangle around the $c$-axis chain is close to the $\mathrm{F}$ or $\mathrm{OH}$ anions which reside at the center of the triangle, the other $\mathrm{Ca}$ site is much further away. Both $\mathrm{Ca}$ sites are rather distant from the anions in $\mathrm{Ca}$ and OA. The $2 s$ and $2 p$ states of $F^{-}$ in FA give contributions which are buried in the upper two bands and lead to no distinctive features. Close examination

\section{Oxygen DOS}

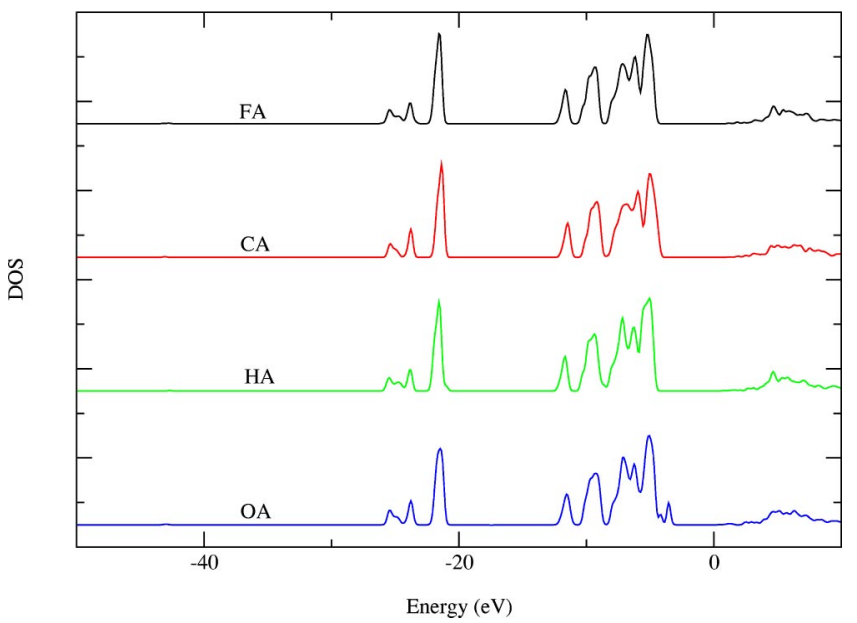

FIG. 4. Oxygen density of states. 


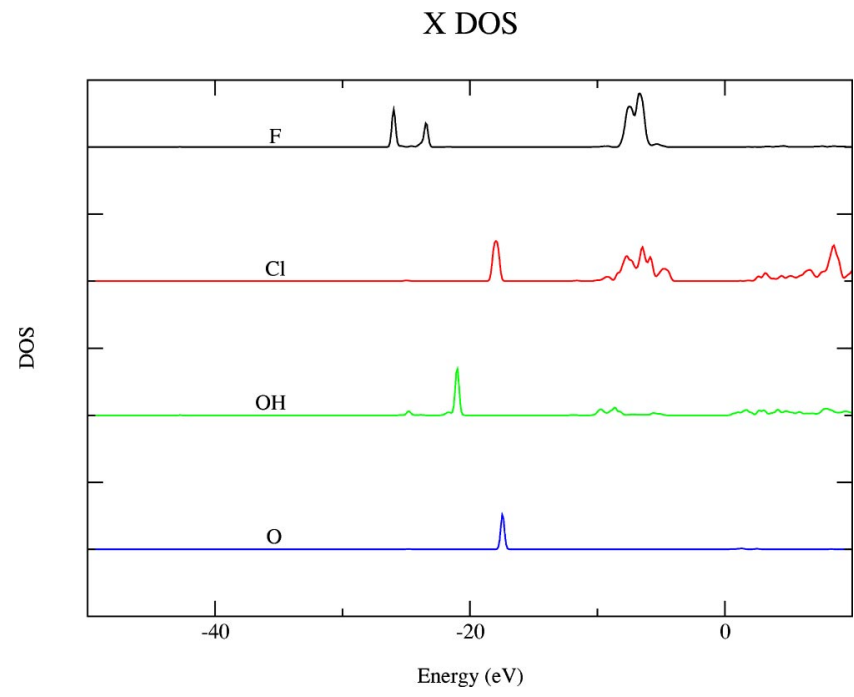

FIG. 5. Density of states of the anions along the $c$ channel.

of the HA DOS shows a small shoulder at the top of the $-23 \mathrm{eV}$ band which is not there for the other apatites. Inspection of Fig. 5 shows that this is due to $\mathrm{OH}^{-}$states. The small peaks at about $-17 \mathrm{eV}$ for both $\mathrm{CA}$ and $\mathrm{OA}$ will be seen to be due to $\mathrm{Cl}^{-}$and $\mathrm{O}^{2-} 3 p$ and $2 p$ states, respectively. We also see that the smaller energy gap for OA is due to $\mathrm{O}^{2-}$ states which encroach into the gap from the top of the valence band. Anions at or close to the calcium triangles seem to contribute only to the already establish bands while anions located in positions far from the calcium triangle contribute also to states in the energy gaps. It is also interesting to note that the DOS may provide a means of differentiating between HA and OA due to the presence of states in the energy gaps of OA which are not present in HA.

\section{D. $\mathrm{Ca}_{5}\left(\mathrm{PO}_{4}\right)_{3}$-an empty $c$ channel}

Calculations have also been performed on the hexagonal system with two $\mathrm{Ca}_{5}\left(\mathrm{PO}_{4}\right)_{3}$ formula units per unit cell which amounts to FA with all the $c$-axis fluorine atoms removed. In the tables and figures this case is referred to as "c empty." This system proved to have a stable total-energy minimum, and is at least metastable. The equilibrium lattice parameters and binding energy given in Table I are similar to the calculated values for the real apatites, although the binding is less than for any of the others. The main features of the density of states shown in Fig. 3 are also similar to those of the apatites except for the presence of states at the Fermi energy and throughout the upper half of the band gap due to the electrons which in the apatites would have been attracted to the $c$-axis chains to form negative ions. The rest of the atomic arrangement is also rather similar to that of the apatites. For example, the sizes of the $\mathrm{Ca}$ and $\mathrm{P}$ triangles lie within the range of values of the other apatites. We conclude that the structures of the apatites are insensitive to the choice of anion in the $c$-axis column, so much so that if these are removed completely the structure is largely unchanged and remains stable, or at least metastable.

\section{CONCLUSIONS}

A study of stoichiometric fluorapatite, chlorapatite, hydroxyapatite, oxyapatite, and the apatite structure with the $c$ channel empty have been performed within the theoretical framework of DFT-LDA and pseudopotentials at zero temperature. The calculations suggest that the hexagonal system is possible in all the studied apatites, even in the case of HA and CA. The monoclinic structure is also possible in HA and $\mathrm{CA}$ and has almost the same total energy per molecule as the hexagonal system. Therefore no relationship between stoichiometry and crystal structure was found. The space groups of FA and OA are in agreement with the experimental results. In the case of CA and HA the space groups do not have the mirror planes which is in some contradiction with the experiments. The calculations also showed that the apatites are quite flexible regarding the presence of different anions in the $c$ channel, which explains why the exchange of anions of different kind is easily performed in apatites.

\section{ACKNOWLEDGMENTS}

This work was supported by a CRD grant of the NSERC of Canada and Millenium Biologix Inc. L.C. also acknowledges W.A. Miller Materials and Manufacturing Ontario Ph.D. Graduate Award. A.R. acknowledges support from DGES, Basque University, and EC-RTN program NANOPHASE (Contract No. HPRN-CT-2000-00167) and COMELCAN (Contract No. HPRN-CT-2000-00128). Discussions with Dr. M. Sayer, Dr. T. Smith, and Dr. X. Yin are gratefully acknowledged.
*Electronic address: calderin@physics.queensu.ca

${ }^{1}$ S. Langstaff, Ph.D. thesis, Queen's University, Kingston, Ontario, Canada, 1999.

${ }^{2}$ J. C. Elliot, Structure and Chemistry of the Apatites and Other Calcium Orthophosphates (Elsevier Science, Amsterdam, 1994).

${ }^{3}$ M. Greenblatt and J. H. Pifer, J. Chem. Phys. 66, 559 (1997).

${ }^{4}$ P. E. Mackie, J. C. Elliot, and R. A. Young, Acta Crystallogr., Sect. B: Struct. Crystallogr. Cryst. Chem. 28, 1840 (1972).

${ }^{5}$ J. C. Elliot, Science 180, 1055 (1973).

${ }^{6}$ H. Morgan, R. M. Wilson, J. C. Elliot, S. E. P. Dowker, and P. Anderson, Biomaterials 21, 617 (2000).

${ }^{7}$ T. Ikoma, A. Yamazaki, S. Nakamura, and M. Akao, J. Solid State
Chem. 144, 272 (1999).

${ }^{8}$ M. I. Kay and R. A. Young, Nature (London) 204, 1050 (1964).

${ }^{9}$ J. C. Trombe and G. J. Montel, J. Inorg. Nucl. Chem. 40, 15 (1978).

${ }^{10}$ P. A. Henning, A. R. Landa-Canovas, A. K. Larsson, and S. Lidin, Acta Crystallogr., Sect. B: Struct. Sci. 55, 170 (1999).

${ }^{11}$ V. Louis-Achille, L. De Windt, and M. Defranceschi, Comput. Mater. Sci. 10, 346 (1998).

${ }^{12}$ Q. Song, C. Wang, and S. Wen, Philos. Mag. A 77, 1309 (1998).

${ }^{13}$ W. Kohn and L. J. Sham, Phys. Rev. 140, A1133 (1965).

${ }^{14}$ W. E. Pickett, Comput. Phys. Rep. 9, 115 (1989); M. C. Payne, M. P. Teter, D. C. Allan, T. A. Arias, and J. D. Joannopoulos, 
Rev. Mod. Phys. 64, 1045 (1992).

${ }^{15}$ N. Troullier and J. L. Martins, Phys. Rev. B 43, 8861 (1991).

${ }^{16}$ D. M. Ceperley and B. J. Alder, Phys. Rev. Lett. 45, 566 (1980).

${ }^{17}$ J. P. Perdew and A. Zunger, Phys. Rev. B 23, 5048 (1981).

${ }^{18}$ P. Ordejón, E. Artacho, and J. Soler, Phys. Rev. B 53, 10441 (1996).

${ }^{19}$ J. M. Soler, E. Artacho, J. D. Gale, A. García, J. Junquera, P.
Ordejón, and D. Sánchez Portal, J. Phys.: Condens. Matter 14, 2745 (2002).

${ }^{20}$ G. Onida, L. Reining, and A. Rubio, Rev. Mod. Phys. 74, 601 (2002).

${ }^{21}$ J. Furthmüller, G. Cappellini, H.-Ch. Weissker, and F. Bechstedt, Phys. Rev. B 66, 045110 (2002). 